\title{
Non-Communicating inactive rudimentary horn of the uterus presenting with dysmenorrhoea-a case report of successful laparoscopic excision
}

\author{
Rachana Dwivedi ${ }^{1}$, Keerthi Perera ${ }^{2}$, Padma Eedarapalli ${ }^{1}$ \\ ${ }^{1}$ The Royal Bournemouth and Christchurch Hospitals NHS Foundation Trust, Bournemouth, UK; \\ ${ }^{2}$ Poole Hospital NHS Foundation Trust, Poole, UK. \\ Email: dwiver28@gmail.com
}

Received 7 September 2011; revised 17 October 2011; accepted 27 October 2011.

\begin{abstract}
Uterine developmental abnormalities can cause both obstetric and gynaecological complications. Non-functioning rudimentary uterine horn is a rare cause of dysmenorrhoea which usually starts after menarche. Cases with lateral/inferior arrangement of blood supply to the functioning rudimentary horn and lapascopic removal have been described in literature. Ours is a unique case of non-functioning rudimentary horn with an unusual medial arrangement of vasculature successfully treated by laparoscopic excision. We also present a brief review of the literature. The patient presented with irretractable dysmenorrhoea despite hormonal manipulation and analgesics. Following the diagnosis using laparoscopy and hysteroscopy and MRI, the left sided non-communicating rudimentary horn with inactive endometrium was removed laparoscopically. Her symptoms were resolved and this was followed by successful pregnancies. Diagnosis of rudimentary horn with inactive endometrium is difficult. Ultrasound is unreliable. MRI, 3D CT scan and 3D ultrasound are gaining popularity. Accurate, prior identification of the type using laparoscopy and hysteroscopy if necessary is essential. This helps in surgical planning. The laparoscopic approach is increasingly being used to resect these horns due to its safety and merits.
\end{abstract}

Keywords: Dysmenorrhoea; Rudimentary Horn; Laparoscopic Excision

\section{INTRODUCTION}

Congenital uterine anomalies result from arrested development of one or both of the Mullerian ducts and/or their defective fusion. In $80 \%$ - $90 \%$ of cases, there is no communicating channel between the two uterine cavi- ties [1]. There are many cases reported with non-communicating rudimentary horn and the functioning endometrium associated with various complications such as dysmenorrhoea, adenomyosis, ectopic pregnancy and haematometra [2,3]. There are also several in literature, treated with laparoscopic excision where the blood supply of the rudimentary horn was either lateral or inferior [4]. However, to our knowledge, there are no case reports of unicornuate uterus and a non-communicating rudimentary horn with an inactive endometrium presenting with unilateral dysmenorrhoea. Moreover, none so far have described a medial arrangement of vasculature.

We report a case of an 18 year old girl who presented with primary cyclic spasmodic dysmenorrhoea which was relieved after laparoscopic excision of type 3 rudimentary horn. The horn was non-communicating with a well developed horn on the other side. The vessels were arranged medially and the endometrium was non-functioning.

\section{CASE REPORT}

The nulliparous girl was first referred to paediatricians at the age of 14 for left loin pain which settled in 2 days. She presented a year later with similar pain which was thought to be due to renal colic. An Ultrasound scan and an X-ray were unremarkable. Two years later she presented to gynaecologists for pelvic pain and cyclical left sided dysmenorrhoea which did not respond to analgesics. Clinical examination elicited tenderness in the left iliac fossa. Repeat ultrasounds and X-Rays were normal and the pain settled on minipill.

She presented again to gynaecologists with cyclical left sided dysmenorrhoea despite minipill, tricycling combined pill and depot provera. On laparoscopy, the uterine fundus appeared broad. The right tube and ovary were normal. The left tube had a small $2 \mathrm{~cm}$ sized swelling near the isthmic end but not continuous with the fundus 
(Figure1). This was confirmed with methylene blue dye test. There was no evidence of endometriosis. A rudimentary uterine horn was suspected. She subsequently had a hysteroscopy which confirmed the fully developed right horn with a single right sided tubal ostium. Magnetic resonance imaging (MRI) further confirmed this finding and detected a $1 \mathrm{~cm}$ sized non-communicating rudimentary horn with endometrial lining on the left side (Figure 2). Renal ultrasound excluded any urinary tract abnormality. She was offered excision of the left horn and fallopian tube laparoscopically. This was carried out successfully through 3 ports using tripolar scissors. The arterial supply coursed medial to the rudimentary horn (Figure 3). The left hemi uterus was only loosely attached to the right sided well developed horn. The left round and utero-ovarian ligaments were diathermied and transected. After identification of the ipsilateral ureter, the uterine vessels on the medial side of this horn (to the left of the right horn) was then similarly cauterised and cut (Figure 4). The patient recovered well from the operation and was discharged home within 24 hours. Histology showed a normal fallopian tube, and the rudimentary uterine structure measured $35 \mathrm{~mm} \times 20 \mathrm{~mm} \times 15 \mathrm{~mm}$ with a lumen lined by inactive endometrium.

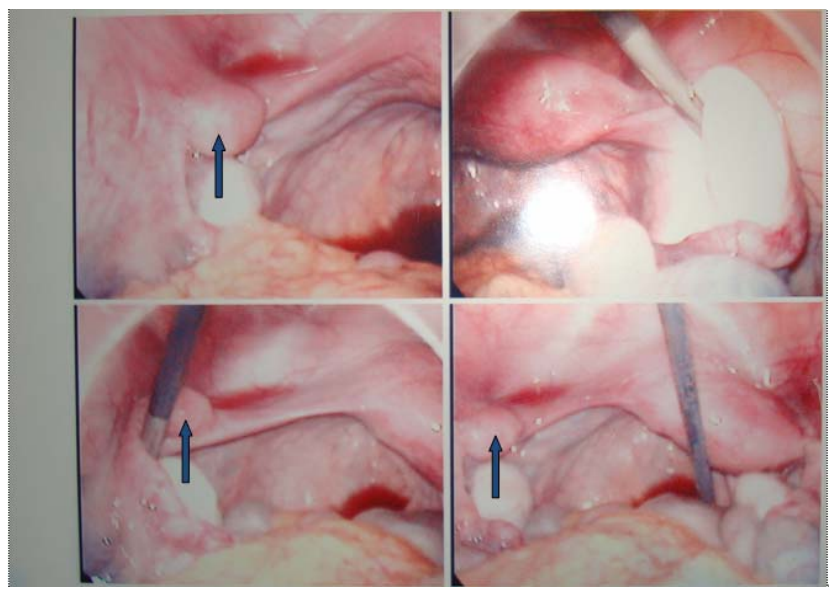

Figure 1. Laparoscopy demonstrating the rudimentary left uterine horn attached to the left fallopian tube.
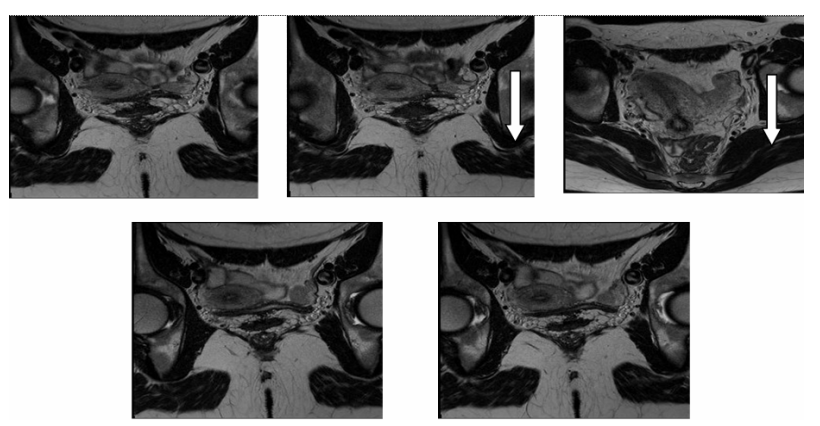

Figure 2. MRI Images of Noncommunicating rudimentary horn.

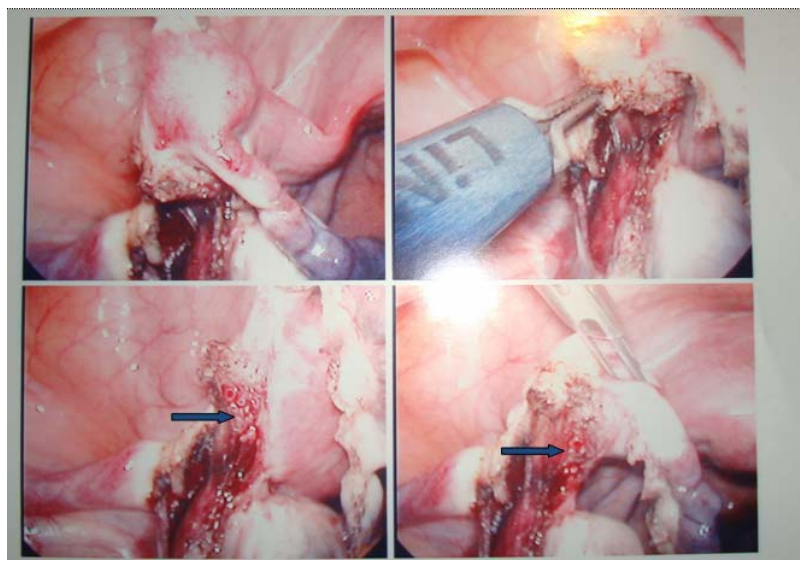

Figure 3. Laparoscopic excision of the left rudimentary horn and fallopian tube demonstrating the medial arrangement of vasculature.

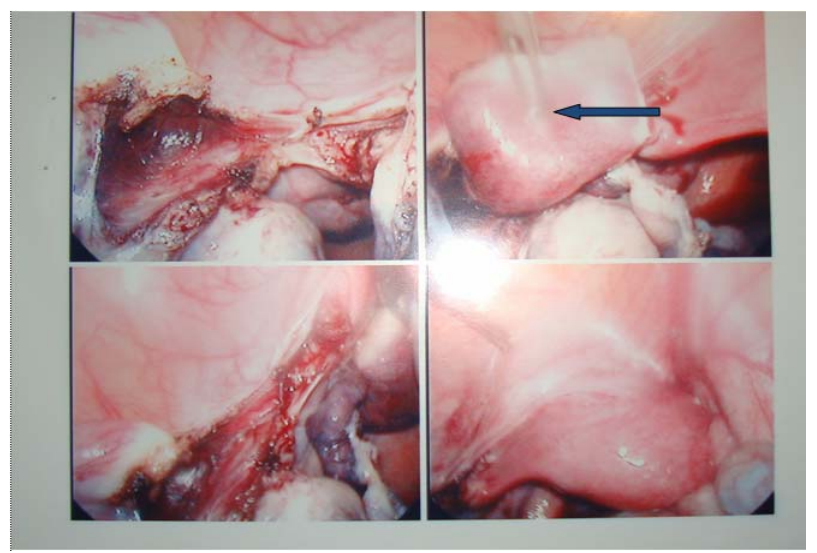

Figure 4. Excised rudimentary horn.

Although her left sided cyclical dysmenorrhoea resolved postoperatively, she continued to have mild cyclical central dysmenorrhoea. She was content to manage this with minipill. Subsequently she had successful pregnancy with an emergency caesarean section for obstetric reasons and is currently pregnant with her second child (at the time of submission of this paper).

\section{DISCUSSION}

The incidence of Mullerian anomalies in the reproductive age group varies from $0.50 \%-017 \%$. The incidence of unicornuate uterus is $2.5 \%-13.2 \%$ [5]. The frequency of rudimentary horn is extremely rare at $1: 100,000$. In $80 \%-90 \%$ cases there is no communication with the other horn. Rudimentary horn could be either firmly attached to the unicornuate uterus or separated by a loose band of tissue. The American Society for Reproductive Medicine (ASRM) [6] divides this into 4 subtypes: Figure 1 unicornuate uterus with a communicating rudimentary horn; Figure $\mathbf{2}$ and $\mathbf{3}$ with a non-communicating horn, with or without cavity and in Figure 4, an iso- 
lated unicornuate uterus.

Women with obstructive uterine anomalies usually present with chronic cyclic or continuous pelvic pain. The presentation can be delayed with increasing severity of dysmenorrhoea, with each subsequent menstrual period as a common feature. The symptoms may be severe enough to interfere with quality of life and may not respond to medication. Unlike in obstructive uterine anomalies where symptoms are caused by the accumulation of blood in the rudimentary horn, disordered arrangement of uterine musculature and abnormal contraction are thought to be the cause in cases such as ours with inactive endometrium [7]. Unequal development of $\mathrm{Mu}-$ llerian system gives rise pain on one side of the pelvis. Torsion of the rudimentary horn has also been reported. Other complications include endometriosis and primary infertility. Anomalies of the urinary system are usually associated with uterine developmental abnormalities due to their close embryological origins. Endometriosis results from retrograde menstruation from the functioning endometrial cavity through the ipsilateral fallopian tube. Obstetric complications such as rupture uterus, recurrent miscarriages, premature labour and malpresentation can also occur [8].

A high index of suspicion for Mullerian duct abnormality is therefore needed in teenagers with dysmenorrhoea to prevent delays in diagnosis [9]. Ultrasonography including hysterosonography is not specific in nonfunctioning uterine horns as the detail of adnexa is insufficient. In a recent review of the literature, the sensitivity of ultrasonography even in cases with functioning horns was only $26 \%$. MRI is much more accurate and detected all cases in these series and is considered to be the gold standard [10]. 3D CT scanning has been used in some cases. 3D ultrasound also may also have a future role. An early recourse to laparoscopy combined with hysteroscopy is required to confirm the diagnosis most cases.

Resection not only offers symptomatic relief in cases unresponsive to medication, it also prevents the possibility of pregnancy in the rudimentary horn due to transperitoneal migration of sperm or fertilised ovum. Accurate diagnosis of the anomaly is required prior to excision to decide on the precise surgical approach as in some cases the two horns are firmly attached. This requires difficult dissection to develop a plane between the hemi uteri. If the other tube is normal, the ipsilateral fallopian tube should be removed to prevent ectopic pregnancy. If the other tube is damaged, microsurgical transposition of the ipsilateral tube may need to be considered [11]. Although laparotomy was used traditionally, there are several publications of the laparoscopic removal of the rudimentary horn [10]. This follows a similar trend in other types of gynaecological surgery. Most cases are amena- ble to laparoscopic management and this is preferred to prevent adhesions and damage to the myometrium of the well developed horn. Rudimentary horn attached to the unicornuate uterus by a band of tissue, as in our case, is relatively easy to dissect. Minimally invasive, with well established safety profile, laparoscopic approach has other advantages such as shorter hospital stay and quicker return to activities [12]. Aberrant blood vessels and urological anomalies increase the risk of bleeding and inadvertent injury. Review of MRI scans with radiologists prior to surgery allows selected cases to be referred to advanced laparoscopic surgeons.

\section{REFERENCES}

[1] Dadhwal, V., Mittal, S., Kumar, S. and Barua, A. (2000) Hematometra in postmenarchal adolescent girls: A report of two cases. Gynecologic and Obstetric Investigation, 50, 67-69. doi:10.1159/000010284

[2] Chakravarthi, S. and Chin, K. (2003) Rudimentary uterine horn: Management of a diagnostic enigma. Acta $\mathrm{Ob}$ stetricia et Gynecologica Scandinavica, 82, 1153-1154. doi:10.1046/j.1600-0412.2003.00234.X

[3] Goel, P., Aggarwal, A., Devi, K., Takkar, N., Saha, P.K. and Huria, A. (2005) Unicornuate with non-communicating rudimentary horn different clinical presentations. The Journal of Obstetrics \& Ganecology of India, 55, 155-158.

[4] Falcone, T., Gidwani, G., Paraiso, M., Beverly, C. and Goldberg, J. (1997) Anatomical variation in rudimentary horns of a unicornuate uterus: Implications for laparoscopic surgery. Human Reproduction, 12, 263-265. doi:10.1093/humrep/12.2.263

[5] Green, L.K. and Harris, R.E. (1976) Uterine anomalies: Frequency of diagnosis and associated obstetric complications. Obstetrics Gynecology, 47, 427-429.

[6] Buttram, V.C. Jr. and Gibbons, W.E. (1979) Mullerian anomalies: A proposed classification (an analysis of 144 cases). Fertility and Sterility, 32, 40-46.

[7] Jeffcoate, N. (2006) Malformation and maldevelopments of the genital tract: In principles of gynaecology. The 4th Edition, Butterworth \& Co Ltd, London, 138.

[8] Atmaca, R., Germen, A.T., Burak, F. and Kafkasli, A. (2005) Acute abdomen in a case with noncommunicating rudimentary horn and unicornuate uterus. Journal of the Society of Laparoendoscpic Surgeons, 9, 235-237.

[9] Badawy, S.Z.A. and Montgomery, S. (2009) Severe primary dysmenorrhoea due to functioning noncommunicatimg rudimentary horn. Journal of Gynecologic Surgery, 25, 23-27. doi:10.1089/gyn.2009.B-02330

[10] Spitzer, R.F., Kives, S. and Allen, L.M. (2009) Case series of laparoscopically resected noncommunicating functional uterine horns. Journal of Pediatric and Adolescent Gynecology, 22, E23-E28. doi:10.1016/j.jpag.2007.12.001

[11] Gold Berg, J.M. and Friedman, C.I. (1988) Microsurgical fallopian tube transposition with subsequent term pregnancy. Fertility and Sterility, 50, 661-662. 
[12] Liatsikos, S.A., Tsikouras, P., Souftas, V., Ammari, A., Prassopoulos, P., Maroulis, G. and Liberis, V. (2010) Diagnosis and laparoscopic management of a rudimentary uterine horn in a teenage girl, presenting with hema- tometra and severe endometriosis: Our experience and review of literature. Minimally Invasive Therapy and Allied Technologies, 19, 241-247.

doi:10.3109/13645701003644491 